\title{
Faecal calprotectin and faecal occult blood tests in the diagnosis of colorectal carcinoma and adenoma
}

\author{
J Tibble, G Sigthorsson, R Foster, R Sherwood, M Fagerhol, I Bjarnason
}

\begin{abstract}
Background and aims-Testing for faecal occult blood has become an accepted technique of non-invasive screening for colorectal neoplasia but lack of sensitivity remains a problem. The aim of this study was to compare the sensitivity and specificity of faecal calprotectin and faecal occult blood in patients with colorectal cancer and colonic polyps.

Methods-Faecal calprotectin and occult blood were assessed in 62 patients with colorectal carcinoma and 233 patients referred for colonoscopy. The range of normality for faecal calprotectin (0.5-10.5 $\mathrm{mg} / \mathrm{l})$ was determined from 96 healthy subjects.
\end{abstract}

Results-Median faecal calprotectin concentration in the 62 patients with colorectal carcinoma (101 mg/1, 95\% confidence interval (CI) 57-133) differed significantly from normal (2.3 mg/1, 95\% CI 1.6-5.0) with $90 \%$ of patients having elevated levels (normal $<10 \mathrm{mg} / \mathrm{l}$ ) whereas only $36 / 62$ $(58 \%)$ had positive faecal occult bloods. There was no significant difference in faecal calprotectin levels when considering location or Dukes' staging of tumour. Percentage positivity of faecal occult bloods was significantly higher for Dukes' stage C and D cancers compared with Dukes' A and $B$. In the colonoscopy group, 29 patients with adenomatous polyps were detected in whom the median faecal calprotectin was $12 \mathrm{mg} / 1$ (95\% CI 2.9-32). Sensitivity for detection of adenomatous polyps was $55 \%$ using the calprotectin method and $10 \%$ using faecal occult blood testing. The overall sensitivity and specificity of calprotectin for colorectal cancer and adenomatous polyps as a combined group was $79 \%$ and $72 \%$, respectively, compared with a sensitivity and specificity of faecal occult blood of $43 \%$ and $92 \%$. Conclusions-Faecal calprotectin is a simple and sensitive non-invasive marker of colorectal cancer and adenomatous polyps. It is more sensitive than faecal occult blood tests for detection of colorectal neoplasia at the cost of a somewhat lower specificity.

(Gut 2001;49:402-408)

Keywords: colorectal cancer; faecal occult blood testing; calprotectin

Colorectal cancer is the second commonest cause of death from malignancy in the Western world. In the USA it accounts for $14 \%$ of cancer deaths with about 134000 colorectal cancer registrations and 55000 deaths each year ${ }^{1}$ while in the UK there are an annual 28000 cancer registrations and 19000 deaths due to this disease. ${ }^{2}$ Survival rates are closely related to the stage of cancer at the time of diagnosis and the most promising approach to reducing mortality rates is early detection of precancerous or cancerous lesions. There is now overwhelming epidemiological evidence and molecular biological data to substantiate previous suggestions of the colonic adenomacarcinoma progression. ${ }^{3-5}$ Collectively, such data have increased the pressure to develop novel approaches for colon cancer detection, critical for secondary prevention through mass population screening whereby early diagnosis of colorectal cancer will detect tumours with the best prognosis and result in improved survival rates.

The most widely accepted non-invasive method for detecting colorectal cancer is faecal occult blood (FOB) testing. Screening in asymptomatic populations have, at best, reduced mortality rates by $15-33 \% .^{6-8}$ There are however many problems with screening using FOB. The sensitivity of the most commonly used guaiac based FOB tests may be as low as $26 \%{ }^{9}$ which means that $74 \%$ of patients with malignant lesions will remain undetected by this method, presumably because blood loss from the tumour may be intermittent or below the detection threshold (2-4 ml of blood/100 g stool). The test may also not be suitable for screening of precancerous adenomas which often do not bleed. ${ }^{10}{ }^{11}$ There are also practical difficulties with certain types of FOB tests which require patients to provide three stool samples while subject to some dietary restrictions. ${ }^{12-14}$

In order to increase the detection rate, clinicians have sought alternative methods for detection of early colorectal cancer/adenomas. A large trial of flexible sigmoidoscopy (compromising the non-invasive nature of investigation) in an asymptomatic population is now underway in the USA ${ }^{15}$ and the $\mathrm{UK}^{16}$ to assess whether this will lead to significantly improved survival for colorectal cancer, but are not expected to yield results until 2008, while flexible sigmoidoscopy is now widely used as an initial examination in symptomatic patients.

Colorectal cancer is associated with a local acute inflammatory reaction so that in some cases it can be visualised by white cell neutrophil scanning. ${ }^{17}$ Calprotectin is a stable neutrophil specific marker which can be assayed in stool with high precision and ease. ${ }^{18}$ Within the neutrophil calprotectin is found in the extra

Abbreviations used in this paper: $\mathrm{FOB}$, faecal occult blood.
Accepted for publication 26 February 2001 
lysosomal cytosol and constitutes up to $60 \%$ of the total protein content. ${ }^{19}$ Levels of faecal calprotectin are increased in patients with colorectal cancer but immunohistochemical examination of colorectal cancer specimens has shown reactivity confined to neutrophilic granulocytes with no reactivity seen in neoplastic cells, ${ }^{20-22}$ suggesting that elevated faecal levels may be due to neutrophil shedding from an ulcerated tumour. The purpose of this study was to assess whether faecal calprotectin is an improvement on the sensitivity and specificity of FOB in current use as a non-invasive biochemical marker for colorectal cancer and colorectal polyps.

\section{Patients and methods}

AIMS

The aims of the study were to (i) assess and compare the sensitivity of faecal calprotectin and FOB for colorectal carcinoma in symptomatic patients with colorectal cancer and relate the results to cancer site or degree of invasion; (ii) assess and compare the sensitivity of the two tests for colorectal polyps subsequently found at colonoscopy; and (iii) assess and compare the specificity of the two tests for detecting colorectal cancer/premalignant polyps.

\section{PATIENTS}

Ninety six healthy volunteers (51 males, 45 females, mean age 41 years) provided single stool samples to obtain a reference range for faecal calprotectin. Controls were predominantly healthcare workers and their relatives, who had no symptoms of gastrointestinal or other diseases, in particular respiratory tract infections, ${ }^{18}{ }^{23}$ on direct questioning. Other exclusion criteria for controls were as for patients studied.

Sixty two consecutive patients (22 females, 40 males; mean age 68 years (range 36-92)) diagnosed with colorectal cancer by barium enema or colonoscopy were recruited to provide faecal samples for calprotectin and occult blood testing; 56/62 (90\%) of these patients underwent operative resection of the tumour and six patients had tumours which were considered inoperable due to advanced metastatic spread.

A total of 233 consecutive patients (130 females, 103 males; mean age 56 years (range 17-91)) referred for colonoscopy for polyp follow up, colorectal cancer surveillance, iron deficiency anaemia, or investigation of colonic symptoms thought to be compatible with or suggestive of colonic neoplasia were also recruited to provide samples for faecal calprotectin and occult blood testing. No patient had a previous diagnosis of colorectal disease except for those undergoing polyp/cancer surveillance where the most recent colonoscopy was at least 12 months before recruitment. All polyps had to have been removed at the previous colonoscopy. Patients taking nonsteroidal anti-inflammatory drugs and those with a high alcohol intake (>14 units/week for females, $>21$ units/week for males) were excluded from the study as these have been shown to cause an enteropathy and raised faecal calprotectin, ${ }^{24}$ and faecal sampling was performed when patients had no evidence of respiratory tract infection. ${ }^{18} 23$

All patients attended a preoperative or precolonoscopic assessment clinic where the nature of the study was explained. Patients provided stool samples prior to surgery/ colonoscopy and those in the colonoscopic group underwent additional investigation if relevant where the cause for symptoms after undergoing colonoscopy were not determined and therefore all patients were given a final diagnosis. All stool samples were provided prior to any administered bowel preparation.

The study was approved by the King's Healthcare local research ethics committee and all patients gave informed consent.

Faecal occult blood tests

For 48 hours prior to collection of samples for FOB testing, all patients were required to observe a red meat free diet, avoid eating raw fruits/vegetables containing peroxidase-like substances such as turnips, broccoli, and parsnips, and avoid ingestion of preparations containing vitamin C. Patients completed a Hema screen card (Immunostics. Inc., 3505 Sunset Ave Ocean, New Jersey 07712, USA), a guaiac based FOB, on three consecutive stool samples which was returned on the day of the last sample and analysed unrehydrated on the day of arrival for FOB. Patients were asked to follow the instructions on the information sheet provided with the test pack.

\section{Faecal calprotectin}

Patients provided a single stool sample for calprotectin measurement and returned it on the same day. Stool samples were frozen on receipt at $-20^{\circ} \mathrm{C}$. After thawing, a single $5 \mathrm{~g}$ aliquot $^{1825}$ was suspended in $10 \mathrm{ml}$ of faecal extraction buffer (Tris buffered isotonic $(150 \mathrm{mM})$ saline, with $10 \mathrm{mM} \mathrm{CaCl}_{2}$, and $0.25 \mathrm{mM}$ thiomersal as an antimicrobial agent, $\mathrm{pH} 8.4$ ) and homogenised for 45 seconds at $20000 \mathrm{rpm}$ with an Ultra Turrax (Ika Werke, Germany) mechanical homogeniser. The homogenates were centrifuged for 15 minutes at $10000 \mathrm{~g}$ at room temperature. The upper portion of the supernatants were pipetted off, frozen, and stored at $-20^{\circ} \mathrm{C}$ until quantitation by ELISA.

Microtitre plates were coated by adding $200 \mu \mathrm{l}$ of an IgG fraction of a rabbit anticalprotectin $^{26}$ diluted 1:2000 in phosphate buffered saline to each well. The plates were covered with mylar foil and kept at $4^{\circ} \mathrm{C}$ until use. Calprotectin standards ${ }^{22} 233.75-60 \mathrm{mg} / 1$ were prepared by diluting purified calprotectin in assay buffer: Tris $(50 \mathrm{mM})$ buffer containing 150 $\mathrm{mM} \mathrm{NaCl}, 0.5 \mathrm{mM} \mathrm{MgCl}, 2.5 \mathrm{mM} \mathrm{KCl}, 0.25$ $\mathrm{mM}$ thiomersol, $0.05 \%$ Tween 20 , and $10 \mathrm{~g} / 1$ bovine serum albumin ( $\mathrm{pH} \mathrm{8.0)}$. Before use the microtitre plates were washed four times in this buffer, less the bovine serum albumin. The frozen faecal extracts were thawed and diluted $1: 20$ and $1: 200$ (and dilutions of 1:400-6400 when required) in the assay buffer. Standards and diluted samples $(100 \mu \mathrm{l})$ were added to the plates in duplicate which were covered and 


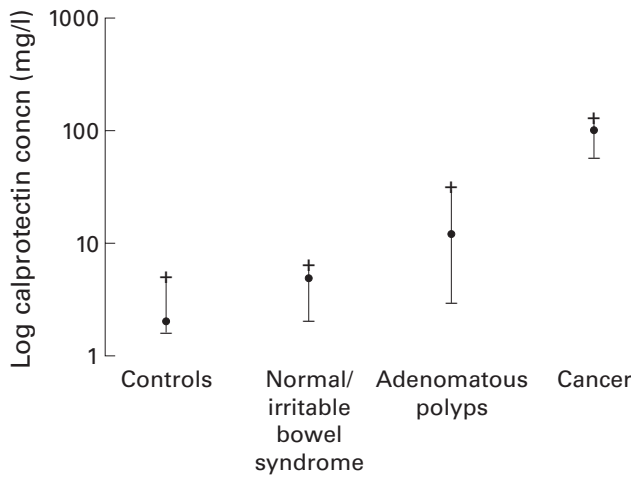

Figure 1 Log faecal calprotectin concentration $(\mathrm{mg} / \mathrm{l})$ in the different diagnostic groups. Median values with $95 \%$ $(+)$ and 5\% (-) confidence intervals are shown. All three diagnostic groups differed significantly from controls (normallirritable bowel syndrome, $p=0.01$; adenomatous polyps, $p=0.0003$; cancer $p<0.0001$ ).

incubated at room temperature for 45 minutes on a plate shaker with an agitating speed of $600 /$ minute. The wells were then washed four times with buffer and $100 \mu \mathrm{l}$ of alkaline phosphatase conjugated anticalprotectin (dilution $1: 1600$ ) was added to each well, the plate covered, and incubated at room temperature for 45 minutes on the plate shaker with an agitating speed of $600 / \mathrm{min}$. Thereafter the wells were washed four times and $100 \mu$ of substrate solution was added $(2 \times 5 \mathrm{mg}$ p-nitro phenolphosphate tablets dissolved in $10 \mathrm{ml}$ of substrate buffer which contained $10 \%$ diethanolamine with $0.5 \mathrm{mM} \mathrm{MgCl}, 0.25 \mathrm{mM}$ thiomersal, $\mathrm{pH}$ adjusted to 9.6 with $\mathrm{HCl}$ ). Optical density (405 nm: measured on a Micro Tracer plate reader; Syva, Milton Keynes, UK) of the highest standard was monitored and the reaction stopped by adding $50 \mu \mathrm{l}$ of a $1 \mathrm{M}$ $\mathrm{NaOH}$ solution to each well when its optical density read between 1.2 and 1.5, similar to the method previously described. ${ }^{18}$

\section{Histopathological assessment}

Each carcinoma specimen obtained at operation was examined histopathologically by the same pathologist and classified according to Dukes' stage and location. Polyps in all cases were obtained by endoscopic snare removal and classified, on the basis of pathology reports, in terms of their histopathological characteristics, size, and location. The locations were classified for both polyps and cancers as rectum, sigmoid, descending, transverse (including hepatic and splenic flexures), and right colon (ascending colon and caecum).

Table 1 Faecal calprotectin levels and percentage of cancers detected by calprotectin and faecal occult blood testing according to Dukes'staging of the tumour

\begin{tabular}{lcccccc}
\hline & \multicolumn{5}{c}{ Calprotectin (mg/l) } & \\
\cline { 3 - 4 } Dukes'stage & $n$ & Median & Range & $\begin{array}{l}\text { Positive by } \\
\text { calprotectin (\%) }\end{array}$ & $\begin{array}{l}\text { Positive by } \\
\text { FOB (\%) }\end{array}$ \\
\hline A: Confined to bowel wall & 10 & 62.5 & $7-933$ & 90 & 20 \\
B: Spread through bowel wall & 24 & 115 & $2-3770$ & 88 & 46 \\
C: Involvement of regional lymph nodes & 14 & 62 & $1.5-314$ & 86 & 100 \\
D: Distant metastases & 14 & 132 & $10.5-3388$ & 100 & 71
\end{tabular}

FOB, faecal occult blood

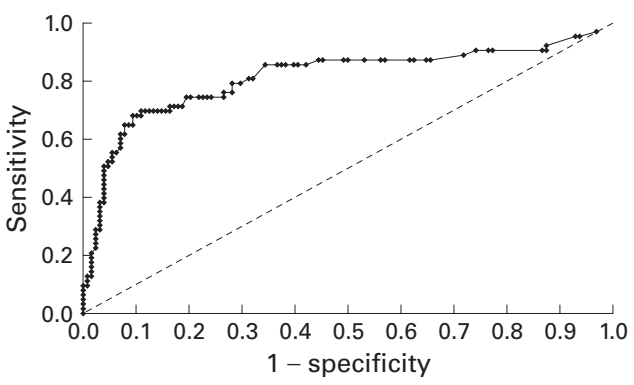

Figure 2 Receiver operating curve for faecal calprotectin in detecting patients with colorectal neoplasia (carcinoma and adenoma). The curve is statistically significant $(p<0.0001)$ with the area beneath it being greater than $50 \%$, and shows the effect of increasing the cut off level for calprotectin on the sensitivity and specificity for detecting colorectal neoplasia.

\section{Statistics}

Statistical analysis of calprotectin levels between groups was performed using the nonparametric Mann-Whitney test, as the results were not normally distributed. The $\chi^{2}$ test was used to compare the percentage positivity of FOB between the different groups. Sensitivities and specificities of the FOB and calprotectin tests were calculated, with those for calprotectin at different cut off values being determined by construction of an ROC curve (plot of sensitivity against 1 -specificity at different levels of calprotectin - see fig 2) including all patients in the colorectal cancer and colonoscopy groups but not those in the control group. The ROC curve enables determination of sensitivity and specificity for disease at different cut off values for a continuous variable. The specificity and sensitivities of the two tests differ depending on the disease in question and we have therefore calculated these for colorectal cancer and adenomatous polyps as a combined group. In our view an ideal screening test detects premalignant disease. Therefore, a positive test in the premalignant group was not regarded as a false positive. All statistics were performed using SPSS for Windows 95.

\section{Results}

The median calprotectin concentration in the 96 normal controls was $2.3 \mathrm{mg} / 1$ (95\% CI $1.6-$ 5.0). The 95 percentile indicates an upper reference limit of $10.0 \mathrm{mg} / \mathrm{l}$ which we have chosen as our cut off value for normality.

Median faecal calprotectin concentration in the 62 patients with colorectal carcinoma (101 $\mathrm{mg} / 1,95 \%$ CI 57-133 mg/l) (fig 1) differed significantly from controls $(\mathrm{p}<0.0001)$. The sensitivity of calprotectin for colorectal cancer $(90 \%)$ was significantly greater $\left(\chi^{2}=26.6\right.$, $\mathrm{p}<0.0001)$ than that for FOB testing $(58 \%)$. There was no significant difference in calprotectin levels $(p>0.2)$ according to the degree of invasion of the tumour using Dukes' staging (table 1) whereas there was a significantly higher positivity rate of FOBs in Dukes' stages C and D compared with Dukes' A and B $\left(\chi^{2}=14.4, p<0.0001\right)$. All patients detected by the FOB test had elevated levels of faecal calprotectin. The location of cancers within the colon was 31 rectal, 21 sigmoid, one descending, four transverse, and five in the right colon. 
There was no significant difference $(\mathrm{p}>0.5)$ in calprotectin values or positivity of FOBs between these different tumour locations.

In the colonoscopy group 51 patients with a total of 86 polyps were detected of whom 22 had hyper/metaplastic (median 2 polyps, range 1-5) and 29 had tubular/tubulovillous adenomas (median 1 adenoma, range $1-3$ ). The range in size of the adenomas was $0.5-5.5 \mathrm{~cm}$ (median 2). Median calprotectin was $4.5 \mathrm{mg} / 1$ (95\% CI 2.9-14) in the hyper/metaplastic group and $12 \mathrm{mg} / 1$ (95\% CI $2.9-32 \mathrm{mg} / \mathrm{l})$ in the adenoma group (fig 1 ) both of which differed significantly from normal $(p=0.003$ and $\mathrm{p}=0.0003$, respectively). There was no significant difference $(p>0.2)$ in calprotectin levels between the different sizes of adenomatous polyps, between adenoma locations (13 proximal to the splenic flexure, 16 distal to the splenic flexure-most distal polyp recorded where more than one present), or between patients with different numbers of polyps. The sensitivity for detecting tubular/tubulovillous adenomas using faecal calprotectin was $55 \%$. The FOBs were positive in only three cases of tubular/tubulovillous adenomas (sensitivity $10 \%)$.

Of the 233 patients who underwent colonoscopy, a final diagnosis of normal or irritable bowel syndrome was made in 128 patients, of whom 109 (85\%) had normal calprotectin levels while $98 \%$ had negative FOBs. Median faecal calprotectin in this group was $5.0 \mathrm{mg} / 1$ (95\% CI 2.0-6.5), which as a group differed significantly from normal controls $(p=0.01)$ (fig 1) but mostly remained well below the cut off value for normality. The remaining 54 patients had a variety of diagnoses (table 2 ). In this group (excluding the four patients with colorectal cancer who are analysed in the cancer group) median faecal calprotectin was 23

Table 2 Diagnoses in the 233 consecutive patients who underwent colonoscopy. The four patients with colorectal cancers are included in the colorectal cancer group when assessing sensitivity of calprotectin and faecal occult blood (FOB) testing for colorectal cancer

\begin{tabular}{|c|c|c|c|c|c|}
\hline \multirow[b]{2}{*}{ Diagnosis } & \multirow[b]{2}{*}{ Total No } & \multicolumn{2}{|c|}{$\begin{array}{l}\text { No with faecal } \\
\text { calprotectin }>10 \mathrm{mg} / \mathrm{l}\end{array}$} & \multicolumn{2}{|c|}{ No with positive FOB } \\
\hline & & No & $\%$ & No & $\%$ \\
\hline Normal/IBS & 128 & 19 & 15 & 3 & 2 \\
\hline Adenomatous polyps & 29 & 16 & 55 & 3 & 10 \\
\hline Hyper/metaplastic polyps & 22 & 6 & 27 & 2 & 9 \\
\hline Colorectal cancer & 4 & 4 & 100 & 4 & 100 \\
\hline Diverticulitis & 24 & 15 & 63 & 3 & 13 \\
\hline Crohn's/ulcerative colitis & 13 & 12 & 92 & 8 & 61 \\
\hline Haemorrhoids & 6 & 0 & 0 & 0 & 0 \\
\hline Angiodysplasia & 4 & 2 & 50 & 1 & 25 \\
\hline Infective diarrhoea & 1 & 1 & 100 & 0 & 0 \\
\hline Pneumatosis coli & 1 & 1 & 100 & 1 & 100 \\
\hline Anal fissure & 1 & 1 & 100 & 0 & 0 \\
\hline
\end{tabular}

IBS, irritable bowel syndrome.

Table 3 Sensitivities and specificities (\%) of faecal calprotectin (at a level of $10 \mathrm{mg} / \mathrm{l}$ ) and faecal occult bloods (FOBs) in the different diagnostic groups

\begin{tabular}{|c|c|c|c|c|}
\hline & \multicolumn{2}{|l|}{ Calprotectin } & \multicolumn{2}{|l|}{ FOBs } \\
\hline & $\begin{array}{l}\text { Sensitivity } \\
(\%)\end{array}$ & $\begin{array}{l}\text { Specificity } \\
(\%)\end{array}$ & $\begin{array}{l}\text { Sensitivity } \\
(\%)\end{array}$ & $\begin{array}{l}\text { Specificity } \\
(\%)\end{array}$ \\
\hline Colorectal cancer & 90 & & 58 & \\
\hline Adenomatous polyps & 55 & & 10 & \\
\hline Colorectal cancer + adenomatous polyps & 79 & 72 & 43 & 92 \\
\hline Significant intestinal disease & 77 & 84 & 39 & 97 \\
\hline
\end{tabular}

$\mathrm{mg} / 1$ (95\% CI 8.5-33.5) which differed significantly from normal $(\mathrm{p}<0.001)$. In these patients $32(64 \%)$ had elevated levels of calprotectin while $13(26 \%)$ had at least one positive FOB.

The sensitivities and specificities of faecal calprotectin and occult bloods in different diagnostic groups are shown in table 3 . When considering the colonoscopy and colorectal cancer groups as a whole, the sensitivity of calprotectin for colorectal cancer and premalignant (adenomatous) polyps (that is, malignant and premalignant disease as a combined group) was significantly greater than for FOB testing $\left(79 \% v 43 \%, \chi^{2}=27.3, \mathrm{p}<0.0001\right)$. The specificity of calprotectin for colorectal cancer and adenomas as a joint group at a cut off level of $10 \mathrm{mg} / \mathrm{l}$ was significantly lower than for FOB testing $\left(72 \%\right.$ v $\left.92 \%, \chi^{2}=5.7, p=0.01\right)$. However, if the sensitivity of calprotectin in identifying patients with significant colorectal disease is considered - that is, all patients with colorectal cancer and those in table 2 except those with haemorrhoids, fissures, and metaplastic polyps - then the sensitivity of calprotectin at a level of $10 \mathrm{mg} / 1$ is $77 \%$ with a specificity of $84 \%$ compared with FOB testing with a sensitivity of $39 \%$ and specificity of $97 \%$.

\section{Discussion}

We have made a direct comparison of FOB tests and faecal calprotectin in detecting colorectal neoplasia. The sensitivity for colorectal cancer using calprotectin was $90 \%$ in comparison with $58 \%$ for FOB testing. This accords well with the findings of Kristinsson and colleagues $^{21}$ who demonstrated a sensitivity of $94 \%$ for colorectal cancer using calprotectin. The $60 \%$ sensitivity of our FOB test is similar to that seen in a number of previous studies ${ }^{1127}$ using guaiac based non-rehydrated FOB tests in symptomatic patients with colorectal cancer. In a recent review assessing the sensitivity of FOB testing in asymptomatic non-referred populations, ${ }^{9}$ Ahlquist found that the overall sensitivity for colorectal cancer was $26 \%$. Sensitivity estimates in large Haemoccult screening trials has been reported to be as high as $81 \%{ }^{8}$ However, these are calculated using an approximation of the prevalence of cancer among test negative screenees based on cancers that present during an arbitrary period of follow up. The poor sensitivity of the guaiac based tests have led a number of authors ${ }^{28-30}$ to recommend the immunological based occult blood tests with higher chemical sensitivity for $\mathrm{Hb}$ (2 mgHb/100 g faeces). ${ }^{31}$ However, because of the greater cost and complexity of the test, their use in screening programmes for colorectal cancer has not been widespread, although in time immunochemical tests for blood may be automated, reducing their complexity considerably.

It is estimated that blood loss of $>20 \mathrm{ml} /$ day is required to achieve $80-90 \%$ positivity of FOB tests ${ }^{32-34}$ and the fluctuating levels of blood loss and intermittent bleeding of cancers compromises the sensitivity of the FOB test. 
Calprotectin, although present in blood, enters the bowel lumen as part of an inflammatory process rather than bleeding from the tumour, as it has been calculated ${ }^{20}$ that blood losses of over $300 \mathrm{ml} /$ day would be required to produce a median calprotectin level of $33 \mathrm{mg} / 1$. Calprotectin is a cytosolic protein in neutrophilic granulocytes and macrophages ${ }^{26} 3536$ and immunohistochemical examination of colorectal cancer specimens has shown calprotectin reactivity confined to such cells with no reactivity in neoplastic cells. ${ }^{22}$ Thus the high levels of calprotectin seen in patients with colorectal carcinoma are likely to be due to polymorphonuclear cell infiltration of the tumour and subsequent shedding into the intestinal lumen. Recruitment of such cells to the tumour is almost certainly due to local production of chemotactic factors possibly produced in response to a breach in the protective mucosal lining. Hence calprotectin sensitivity for colorectal cancer is not likely to be affected significantly by variations in tumour blood loss, and may account for the non-significant difference in faecal calprotectin levels between the different Dukes' stages of cancer, results which would need to be confirmed in an asymptomatic population. Patterns of bleeding may significantly affect the sensitivity of FOB tests and may explain why we observed higher sensitivities for Dukes' stages C and D than for stages A and B. Similar observations were made by Frommer et al who found improved sensitivity for Dukes' A tumours compared with Dukes' D when using non-rehydrated Hamoccult II. ${ }^{28}$ St John et al demonstrated sensitivities of $63.6 \%$ for stage $\mathrm{A}$ and $\mathrm{B}$ tumours compared with $89.7 \%$ for stages C and $\mathrm{D}$ when using the HaemoQuant $\mathrm{FOB}^{37} \mathrm{~A}$ logical explanation for FOB sensitivity differing according to stage of disease might be that more advanced tumours bleed more consistently and to a greater extent, thereby improving the number of positive FOB tests. However, in the study of St John et al assessing levels of blood loss in patients with colorectal cancer, mean daily blood loss was dependent only on site of tumour (right sided greater than left) and was not affected by tumour stage. ${ }^{37}$

It may be argued that comparison between faecal calprotectin and FOBs is unfair based on awareness of the inherent problems associated with guaiac based tests, in particular their lower sensitivities for rectal and right sided tumours, ${ }^{7}$ especially as $50 \%$ of our tumours were rectal. Our objective however was simply to compare the two different stool tests (calprotectin $v$ FOB) for the detection of colorectal cancer, irrespective of location. It is of interest that despite the accepted inherent limitations of the FOB tests it has been proposed to use them in a national screening programme for colorectal cancer in the UK.

We have demonstrated a marked difference in the sensitivity of calprotectin and FOB for colorectal adenomas- $55 \%$ versus $10 \%$, respectively. The limitations of FOBs are even more marked when screening for these premalignant lesions where quantitative blood loss is even less and more intermittent than for carcinomas. A number of authors ${ }^{38-41}$ have reported negative Haemoccult reactions in $>75 \%$ of patients with endoscopically proved adenomas, while MacRae and St John ${ }^{11}$ found that only polyps larger than $2 \mathrm{~cm}$ produced consistent detectable bleeding.

To maximise the mortality benefits of colorectal cancer screening it will be necessary to detect and treat adenomatous lesions, which may result in a fall in colorectal cancer incidence, as well as detecting early malignant lesions with the best prognosis. In our study the overall sensitivity of calprotectin for colorectal cancer and adenomatous polyps was 79\% compared with $43 \%$ for FOB testing, accepting that this is in a symptomatic group of patients possibly overestimating the sensitivity values of both tests. The reduction in colorectal cancer mortality seen in the University of Nottingham Minnesota trials (15-33\%, respectively $)^{6-8}$ using guaiac based FOB screening were due to detection of cancers at an earlier stage and not due to the detection of premalignant lesions. The colorectal cancer incidence was the same in both the screened and non-screened groups. If we consider case control studies using sigmoidoscopy which detects both cancers and adenomas, ${ }^{42-45}$ they have demonstrated a $50 \%$ reduction in the risk of developing colorectal cancer and a $59 \%$ reduction in mortality from cancers reached by the sigmoidoscope. Thus with the much higher overall sensitivity of calprotectin for colorectal cancer/adenomatous polyps it has the potential for a greater reduction in mortality than that provided by the currently recommended FOB tests.

If calprotectin is to be considered for use in large screening programmes the issue of specificity has to be addressed. Sensitivity and specificity have an inverse relationship-the sensitivity of the calprotectin test may be improved by lowering the cut off point but at the cost of a lowered specificity for the disease in question. The specificity of the test has important implications on the overall cost of a screening programme. From our study we can only make an assessment of specificity in a group of patients most of whom had symptoms of colorectal disease and were therefore more likely to have intestinal inflammation from a variety of causes than an asymptomatic population. The comparative specificities of calprotectin and FOBs for detecting colorectal cancer and adenomatous polyps as a combined group were $72 \%$ and $92 \%$. By analysis of the receiver operating curve (fig 2), the specificity of the calprotectin test could be improved to $80 \%$ with only a small reduction in the sensitivity (75\%) using a cut off level of $15 \mathrm{mg} / \mathrm{l}$. Such an exercise would need to be performed when assessing the calprotectin assay in an asymptomatic population in order to determine the "best" cut off value, depending on the relative importance given to sensitivity and specificity. If of equal importance then the value which gives the greatest sum of these two parameters should be chosen.

However, if we consider the specificities for detecting all significant colorectal disease 
(table 2 diagnoses, except haemorrhoids, fissures, and hyper/meta plastic polyps), values increase to $84 \%$ and $97 \%$ with sensitivities of $77 \%$ and $39 \%$ for calprotectin and FOBs, respectively. Due to the nature of calprotectin it is likely that it will detect patients with significant inflammation within the gastrointestinal tract, including those with inflammatory bowel disease, diverticulitis, and small bowel enteropathies, explaining the levels of calprotectin seen in such patients in our study. Although screening programmes for colorectal cancer are not intended to detect such conditions, they are often significant diseases for which effective treatment is available and therefore identifying such patients who may benefit from colonoscopic assessment could be regarded as a positive aspect of the calprotectin test. Further evaluation of the sensitivity and specificity of faecal calprotectin will need to be performed in asymptomatic non-referred populations but it may be that in such a group there would be fewer patients with inflammatory conditions and the specificity of calprotectin for colorectal cancer/adenomas might be higher than the $72 \%$ seen in our study.

Compliance is an important factor to take into account when considering the use of a test in a screening programme. Compliance rates for FOB screening in trials each offering screening to over 10000 subjects were on average $63 \%,{ }^{46}$ ranging from $35 \%$ to $92 \% .{ }^{47}{ }^{48}$ The sensitivity of FOB testing is dependant on participants providing three stool samples and it would seem probable that compliance rates would be improved if only one sample was required as is the case for faecal calprotectin. ${ }^{18}$ In addition, compliance of screening for colorectal cancer in asymptomatic high risk groups using colonoscopy may be improved if patients are prescreened with faecal calprotectin whereby an elevated level may reinforce the need for a screening colonoscopy.

In summary, faecal calprotectin appears to be a simple non-invasive surrogate marker of inflammation in patients with colorectal cancer/adenomatous polyps. It has a number of significant advantages over FOB testing in the detection of colorectal neoplasia, most notably a sensitivity of $79 \%$ compared with $43 \%$ when considering malignancy and polyps as a whole. Improved sensitivity however comes at the cost of diminished specificity which has cost implications because of additional investigation. The faecal calprotectin method seems to be a useful adjuvant to the investigation of patients at high risk for colorectal neoplasia but its value in a truly asymptomatic population such as those who would be considered for entry into a national screening programme for colorectal cancer needs to be determined.

Dr Tibble was funded by the NHS Executive South Thames Regional Office, grant No RDF 039.

Conflict of interest. Professor Fagerhol shares patent rights on calprotectin.

1 American Cancer Society. Cancer facts and figures. Atlanta: American Cancer Society, 1996.

2 Hart AR, Wicks AC, Mayberry JF. Colorectal cancer screening in asymptomatic populations. Gut 1995;36:590 8.
3 Muto T, Bussey HJ, Morson BC. The evolution of cancer of the colon and rectum. Cancer 1975;36:2251-70.

4 Morson B. President's address. The polyp-cancer sequence in the large bowel. Proc R Soc Med 1974;67:451-7.

5 Stryker SJ, Wolff BG, Culp CE, et al. Natural history of untreated colonic polyps. Gastroenterology 1987;93:100913.

6 Kronborg O, Fenger C, Olsen J, et al. Randomised study of screening for colorectal cancer with faecal-occult-blood test. Lancet 1996;348:1467-71.

7 Hardcastle JD, Chamberlain JO, Robinson MHE, et al. Randomised controlled trial of faecal-occult-blood screening for colorectal cancer. Lancet 1996;348:1472-7.

8 Mandel JS, Bond JH, Church TR, et al. Reducing mortality from colorectal cancer by screening for fecal occult blood. Minnesota Colon Cancer Control Study. $N$ Engl f Med 1993;328:1365-71.

9 Ahlquist DA. Fecal occult blood testing for colorectal cancer: Can we afford to do this? Gastroenterol Clin North Am 1997;26:41-55.

10 Herzog P, Holtermuller KH, Preiss J, et al. Fecal blood loss in patients with colonic polyps: A comparison of measurements with (51)chromium-labeled erythrocytes and with the Haemoccult test. Gastroenterology 1982;83:957-62.

11 MacRae FA, St John DJB. Relationship between patterns of bleeding and Hemoccult sensitivity in patients with colorectal cancers or adenomas. Gastroenterology 1982;82: 891-8.

12 MacRae FA, St John DJB, Caligiore P, et al. Optimal dietary conditions for Hemoccult testing. Gastroenterology 1982;82: 899-903.

13 Jaffe RM, Kasten B, Young DS, et al. False-negative stool occult blood tests caused by ingestion of ascorbic acid (vitamin C). Ann Intern Med 1975;83:824-6.

14 Jaffe RM, Zierdt W. A new occult blood test not subject to false-negative results from reducing substances. $\mathcal{F}$ Lab Clin Med 1979;93:879-86.

15 Institute NC. Concept approval granted to trial of prostate, lung, colorectal and ovarian screens. Cancer Lett 1989;15: $1-3$.

16 Atkin WS. Flexible sigmoidoscopy as a mass screening tool. Eur $\mathcal{F}$ Gastroenterol Hepatol 1998;10:219-23.

17 Saverymuttu SH, Maltby P, Batman P, et al. False positive localisation of indium-111 granulocytes in colonic carcinoma. Br f Radiol 1986;59:773-7.

18 Roseth AG, Fagerhol MK, Aadland E, et al. Assessment of the neutrophil dominating protein calprotectin in feces. A the neutrophil dominating protein calprotectin in feces. A
methodologic study. Scand $\mathcal{7}$ Gastroenterol 1992;27:793-8.

19 Fagerhol MK, Andersson KB, Naess-Andresen CF, et al. Calprotectin (the L1 leukocyte protein). In: Smith VL, Dedman JR, eds. Stimulus response coupling: the role of intracellular calcium-binding proteins. Boca Raton, Florida: CRC Press Inc, 1990:187-210.

20 Gilbert JA, Ahlquist DA, Mahoney DW, et al. Fecal marker variability in colorectal cancer: calprotectin versus hemoglobin. Scand F Gastroenterol 1996;31:1001-5.

21 Kristinsson J, Roseth A, Fagerhol MK, et al. Fecal calprotectin concentration in patients with colorectal carcinoma. Dis Colon Rectum 1998;41:316-21.

22 Roseth AG, Kristinsson J, Fagerhol MK, et al. Faecal calprotectin: a novel test for the diagnosis of colorectal cancer? Scand $\mathcal{F}$ Gastroenterol 1993;28:1073-6.

23 Meling TR, Aabakken L, Roseth A, et al. Faecal calprotectin shedding after short-term treatment with non-steroidal anti-inflammatory drugs. Scand $\mathcal{F}$ Gastroenterol 1996;31: 339-44.

24 Tibble JA, Sigthorsson G, Foster R, et al. High prevalence of NSAID enteropathy as demonstrated by a simple faecal test. Gut 1999;45:362-6.

25 Tibble JA, Teahon K, Roseth A, et al. A simple method for assessing intestinal inflammation in Crohn's disease. Gut 2000;47:506-13.

26 Dale I BP, Fagerhol MK, Scott H. Distribution of a new myelomonocytic antigen $(\mathrm{L}-1)$ in human peripheral blood myelomonocytic antigen (L-1) in human peript
leukocytes. Am $\mathcal{F}$ Clin Pathol 1985;84:24-34.

27 Allison JE, Feldman R, Tekawa IS. Hemoccult screening in detecting colorectal neoplasm: Sensitivity, specificity, and predictive value: Long-term follow-up in a large group practice setting. Ann Intern Med 1990;112:328-33.

28 Frommer DJ, Kapparis A, Brown MK. Improved screening for colorectal cancer by immunological detection of occult blood. BMF 1988;296:1092-4.

29 St John DJB, Young GP, Alexeyeff MA, et al. Evaluation of new occult blood tests for detection of colorectal neoplasia. Gastroenterology 1993;104:1661-8.

30 Robinson MHE, Marks CG, Farrands PA, et al. Screening for colorectal cancer with an immunological faecal occult for colorectal cancer with an immunological faecal occul

31 Frommer D, Kapparis A. Faecal occult blood testing. Lancet 1983;ii:738

32 Morris DW, Hansell JR, Ostrow JD, et al. Reliability of chemical tests for fecal occult blood in hospitalized patients. Am f Dig Dis 1976;21:845-52.

33 Stroehlein JR, Fairbanks VF, McGill DB, et al. Hemoccult detection of fecal occult blood quantitated by radioassay. Am f Dig Dis 1976;21:841-4.

34 Doran J, Hardcastle JD. Bleeding patterns in colorectal cancer: The effect of aspirin and the implications for faecal occult blood testing. Br f Surg 1982;69:711-13.

35 Fagerhol MK, Dale I, Anderson I. Release and quantitation of a leucocyte derived protein (L1). Scand $\mathcal{f}$ Haematol $1980 ; 24: 393-8$ 
36 Steinbakk M, Naess Andresen CF, Lingaas E, et al. Antimicrobial actions of calcium binding leucocyte L1 protein, calprotectin. Lancet 1990;336:763-5.

37 St John DJB, Young GP, McHutchinson JG, et al. Comparison of the specificity and sensitivity of Haemoccult and HaemoQuant in screening for colorectal neoplasia. Ann Intern Med 1992;117:376-82.

38 Winawer SJ. Screening for colorectal cancer: An overview. Cancer Philadelphia 1980;45:1093-8.

39 Crowley ML, Freeman LD, Mottet MD, et al. Sensitivity of guaiac-impregnated cards for the detection of colorectal neoplasia. F Clin Gastroenterol 1983;5:127-30.

40 Eddy DM. Computer assisted evaluation of screening programs. Washington: US Department of Health, Education, and Welfare, 1979.

41 Lipshutz GR, Katon RM, McCool MF, et al. Flexible sigmoidoscopy as a screening procedure for neoplasia of the colon. Surg Gynecol Obstet 1979;148:19-22.

42 Selby JV, Friedman GD, Quesenberry CP Jr, et al. A case-control study of screening sigmoidoscopy and mortality from colorectal cancer. N Engl f Med 1992;326: 653-7.

3 Winawer SJ, Flehinger BJ, Schottenfeld D, et al. Screening for colorectal cancer with fecal occult blood testing and sigmoidoscopy. $\mathcal{F}$ Natl Cancer Inst 1993;85:1311-18.

44 Muller AD, Sonnenberg A. Protection by endoscopy against death from colorectal cancer: A case-control study among veterans. Arch Intern Med 1995;155:1741-8.

45 Muller AD, Sonnenberg A. Prevention of colorectal cancer by flexible endoscopy and polypectomy. A case-control study of 32702 veterans. Ann Intern Med 1995;123:90410.

46 Van Dam J, Bond JH, Sivak MV Jr. Fecal occult blood screening for colorectal cancer. Arch Intern Med 1995;155: 2389-402.

47 Morris JB, Stellato TA, Guy BB, et al. A critical analysis of the largest reported mass fecal occult blood screening pro-
gram in the United States. Am f Surg 1991;161:101-6.

48 Zoubek V, Zoubkova H. Results of screening for colorectal carcinoma in the district of Bruntal using the Haemoccult test in 1985-1988. Czech Med 1990;13:2-3. 